\title{
The Surprisingly Complex Lives of Massive Galaxies
}

\author{
Rachel Bezanson ${ }^{* \dagger}$ \\ Hubble Fellow \\ Steward Observatory, University of Arizona \\ E-mail: rbezanson@email.arizona.edu
}

\begin{abstract}
Once thought to be relics of a much earlier epoch, the most massive local galaxies are red and dead ellipticals, with little ongoing star formation or organized rotation. In the last decade, observations of their assumed progenitors have demonstrated that billions of years ago, massive galaxies were more compact and morphologically different, possibly with more disk-like structures. The details of this observed evolution can place constraints on the physical processes that have driven massive galaxy evolution through cosmic time.

I will present an overview of current observational studies of the structural and dynamical properties of high-redshift galaxies, focusing on our understanding of how massive galaxies grow through cosmic time. In particular, I will highlight results from the upgraded WFC3 camera on HST, which allows for high-resolution imaging and grism spectroscopy in the rest-frame optical out to $z \sim 3$. Additionally, I will describe results from deep spectroscopic studies, which have provided more detailed information about the stellar populations and dynamics of the high-redshift progenitors of massive galaxies today.
\end{abstract}

Frank N. Bash Symposium 2013: New Horizons in Astronomy

October 6-8, 2013

Austin, Texas

\footnotetext{
*Speaker.

$\dagger$ Support for this work was provided by NASA through Hubble Fellowship grant \# HST-HF-51319.01-A awarded by the Space Telescope Science Institute, which is operated by the Association of Universities for Research in Astronomy, Inc., for NASA, under contract NAS 5-26555.
} 


\section{Introduction}

The deepest images of the night sky, taken by the Hubble Space Telescope (e.g. HUDF [26], XDF [24]) demonstrate the remarkable diversity and preponderance of galaxies in the distant Universe. In the coarsest sense, the primary goal of the study of galaxy evolution is to describe and explain the formation and evolution of these structures through cosmic time. In practice, from an observational standpoint, this involves three primary tasks: the identification of galaxies at different epochs, examination of the properties of those galaxies, and finally the connection of populations of galaxies as they evolve through time. In the past couple of decades, the study of high-redshift galaxies has evolved dramatically; observational surveys are beginning to probe billions of years of the history of the Universe - even glimpsing some of the youngest galaxies at cosmic dawn.

\section{A very brief history of high-z galaxy surveys}

\subsection{Early galaxy redshift surveys}

Much of the progress in the field has been driven by a combination of technological advances and innovative approaches to the identification and study of high redshift galaxies. Early redshift surveys, e.g. the CfA redshift survey [16], spectroscopically targeted all objects above magnitudethresholds to measure the overall distribution of galaxies, and therefore large-scale structure, in the local universe with extreme completeness. More recently the Sloan Digital Sky Survey [1] (SDSS) has provided photometric and spectroscopic information for similarly complete samples of galaxies in swaths of the sky - dramatically shaping our understanding of the properties of local galaxies.

Ideally, as galaxy studies probe greater distances, and therefore longer baselines in time, we would repeat this "brute force" approach to collecting samples of galaxies throughout cosmic time. However, this methodology becomes increasingly unfeasible with redshift as distant galaxies have decreasing space densities, appear fainter, and the majority of their light gets shifted to redder wavelengths. Large, magnitude-limited (and therefore complete for massive galaxies) galaxy surveys taken with multi-object, optical spectrographs exist out to $z \sim 1$ (e.g. DEEP/DEEP2 surveys $[33,62]$ and the PRIMUS survey [14]). These surveys are much less wide-field than local surveys and therefore probe narrower ranges in environments than the SDSS, for example, however they yield a fairly complete census of galaxy properties at these redshifts.

The innovation that fundamentally transformed high-z galaxy studies was moving away from the reliance on spectroscopic redshifts to estimating redshifts using galaxy colors alone. These photometric redshifts rely on using a combination of a number of carefully chosen colors to identify strong features in the spectral energy distributions (SEDs) of galaxies. Generally these photometric redshifts identify breaks (e.g. Lyman or Balmer/4000 $\AA$ breaks) in the galaxy spectra. Perhaps the most notable initial example is that of the discovery of Lyman Break Galaxies (LBGs) [e.g. 42]. These strongly star-forming galaxies can be easily identified by a combination of a red color shortward and a blue color longward of the rest-frame $912 \AA$ Lyman limit. At a given redshift, one can estimate boxes in color-color space in which LBGs will be found. With this technique, large numbers of a specific type of high redshift galaxies can be identified with limited optical photometric data. Furthermore, LBGs in particular are quite bright at a given redshift, so they are ideal for spectroscopic follow-up and confirmation of the particular selection method. Similar 
techniques have been designed to catch other spectroscopic features with both narrow-band filters to catch specific emission lines (e.g. Lyman Alpha Emitters [23]), or broadband photometry in the optical and near-IR (e.g. distant red galaxies, DRGs [20, 58]).

All of these photometric surveys relied on identifying specific types of galaxies, which had very direct consequences. First, the galaxy samples were found and named, generally with an acronym, based on their identification method, not based on their physical properties. This resulted in a somewhat piecemeal understanding of the overall galaxy population at a given redshift. Instead, our understanding of high-z galaxies was an alphabet soup of galaxy samples. The lack of a general census of galaxies rendered drawing connections between galaxy populations at a given epoch or through time extremely difficult.

The next step in improving photometric identification of high-redshift galaxies was the development of medium-band width filters. By using a large number of filters, one can trace out the shape of a galaxy's SED, yielding a very low-resolution spectrum, from which redshift can be estimated. The spectral "resolution" of the sampling of the SEDs determines the accuracy of the photometric redshifts. The first survey to pioneer this approach was the COMBO-17 survey [5], which utilized 17 medium band filters to measure photometric redshifts for $10^{5}$ galaxies. This survey included galaxies of all types and therefore yielded a much more comprehensive view of galaxies in the $z \lesssim 1$ universe. Concurrently, technological advances in near-IR detectors allowed observers to capture the rest-frame optical emission from galaxies out to much higher redshifts. Many groups underwent large photometric surveys designed to identify high-z galaxies based on their SEDs (e.g. FIRES [18] and MUSYC [40] surveys).

\subsection{State of the art in galaxy surveys}

Currently, the pinnacle of high redshift galaxy studies involve a combination of very deep photometric data, particularly focused on longer wavelength light, which also covers a large wavelength range to best sample galaxy SEDs. This has yielded a handful of designated areas on the sky that are well studied across the electromagnetic spectrum from X-ray to radio wavelengths. Several groups have added deep imaging in near-IR medium band filters specifically designed to carefully sample the Balmer/4000 $\AA$ breaks at $z \sim 2-3$ (e.g. NMBS [66] and Z-FOURGE [45] surveys). Although galaxies at these redshifts are quite faint and would require $\sim 10 s$ of hours to follow-up spectroscopically on large telescopes, photometric redshifts that include medium-band photometry have been shown to have excellent accuracy $(\Delta z /(1+z) \sim 1-2 \%)$.

As the rest-frame optical galaxy emission redshifts into the Near-IR, observations in this band have become crucial. Unfortunately, the Earth's atmosphere introduces a fair amount of absorption and variability. Therefore, observations from space, particularly made by the recently upgraded WFC3 camera on the Hubble space telescope have become increasingly important. Currently there are two complementary large galaxy surveys being conducted using HST: CANDELS [21, 25], which provides extremely deep imaging and 3D-HST [11], which adds low-resolution grism spectroscopy. Finally, at $z>13 \mathrm{D}-\mathrm{HST}$ provides a spectroscopic survey of galaxy properties at these redshifts without any preselection biases. In the near future, with the introduction of excellent quality multi-object Near-IR spectrographs on large telescopes will allow for large, higher resolution spectroscopic surveys (such as the forthcoming MOSFIRE Deep Evolution Field (MOSDEF) survey with MOSFIRE on Keck I). 

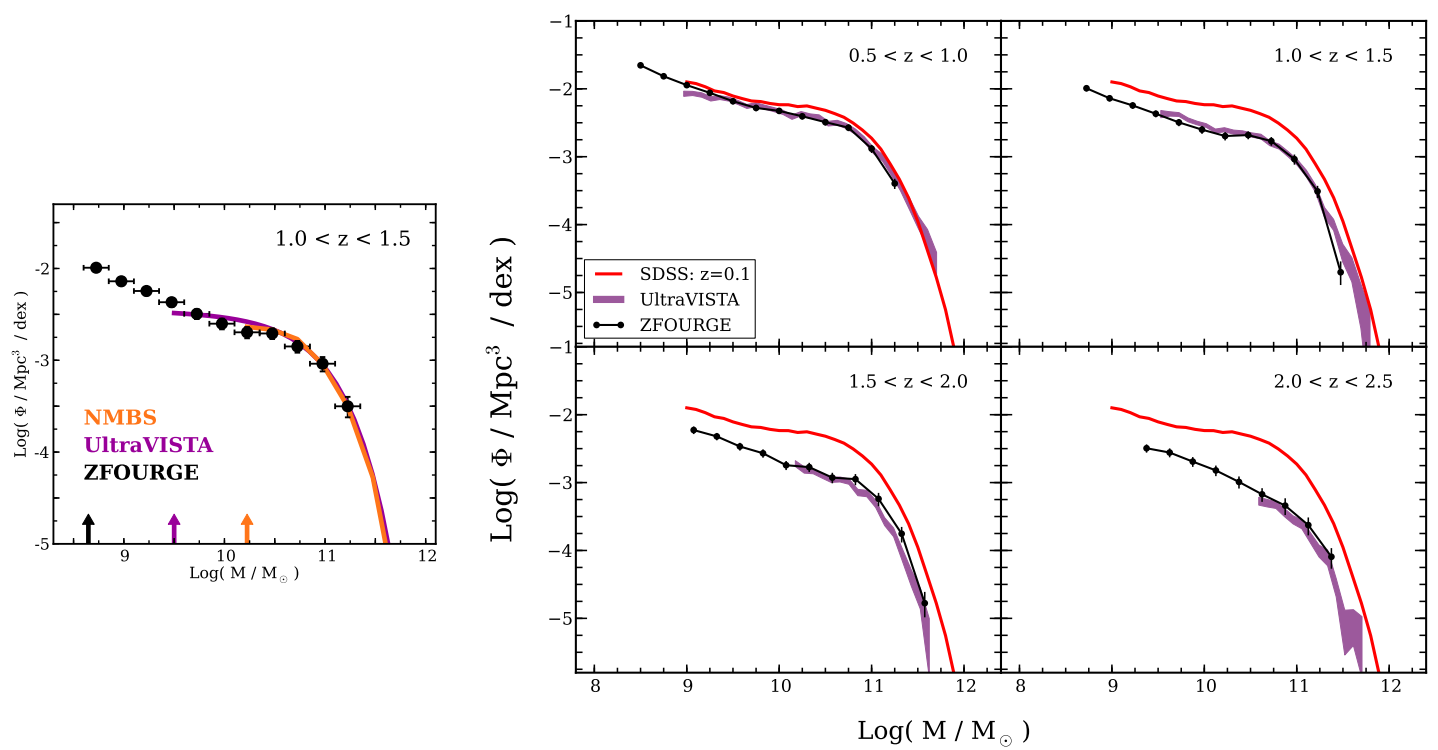

Figure 1: Stellar mass functions from the Z-FOURGE survey (from Tomczak et al. [48]). Left: Stellar mass functions above cited completeness limits (vertical arrows) in three extragalactic surveys at $z \sim 1.25$. Right: Redshift evolution of the stellar mass functions from the Z-FOURGE survey (black) relative to the local stellar mass functions from the SDSS (red). Even at the highest redshift, the Z-FOURGE survey is mass complete to $\log M_{*} \gtrsim 9.25$, which is almost comparable to the SDSS.

\subsection{Vast improvement in survey depths}

Although surveys and complete studies of galaxy evolution were once limited to the most massive galaxies, the latest generation of deep pencil beam surveys are now probing to masscompleteness levels of local galaxy surveys (such as the SDSS). One such survey is the Z-FOURGE survey (observing finished in 2013), which has already published mass functions to unprecedented mass limits (Figure 1, [48]). No longer restricted to follow only the most massive galaxies through cosmic time, some very recent studies have begun to examine the growth and formation of Milky Way mass galaxies [e.g. 38, 61].

\section{Evolution of Massive Galaxies}

The ultimate goal of these large galaxy surveys is not just to identify distant galaxies, but to rather to follow coherent populations of progenitors and descendants through cosmic time. Although photometric galaxy surveys are more comprehensive than early high-z studies, they will still only give a complete description of the galaxy population down to a magnitude limit. Since the observed magnitude of a galaxy is a observational, and not intrinsic property, this can be translated to a more physical mass limit down to which a survey is complete. For the remainder of this article, I will focus on the massive $\left(M_{\star} \gtrsim 10^{11} M_{\odot}\right)$ galaxy population, which, until very recently, has been at the edge of galaxies that can be fully sampled by large surveys out to the highest redshifts.

\subsection{Massive galaxies today}

Massive galaxies in the local universe are generally giant ellipticals. In addition to being 
massive, these galaxies are physically extended, with half-light radii of $r_{e} \gtrsim 5 \mathrm{kpc}$. The stellar populations are primarily old, red and dead, with very little cold gas or on-going star-formation. In addition to their old stellar populations, massive elliptical galaxies are characterized by their dynamically relaxed stellar bodies. Structurally, they are generally quite smooth and follow roughly de Vaucouleur $\left(\rho \propto r^{1 / 4}\right)$ profiles [17] and are dispersion supported.

All of these pieces of evidence suggest that massive galaxies are largely relics of a much earlier time in the history of the Universe. If this were indeed the case, one would predict that the progenitors of elliptical galaxies would remain largely unchanged in observations since the much earlier epoch when their stellar bodies were formed or assembled. Because the stellar populations of the most massive local ellipticals are extremely old ( $>1-10 \mathrm{Gyrs})$, naively we would expect observations of massive galaxies at $z \sim 2$, or roughly 10 billion years in the past, to look structurally similar, with bluer colors due to younger stellar populations.
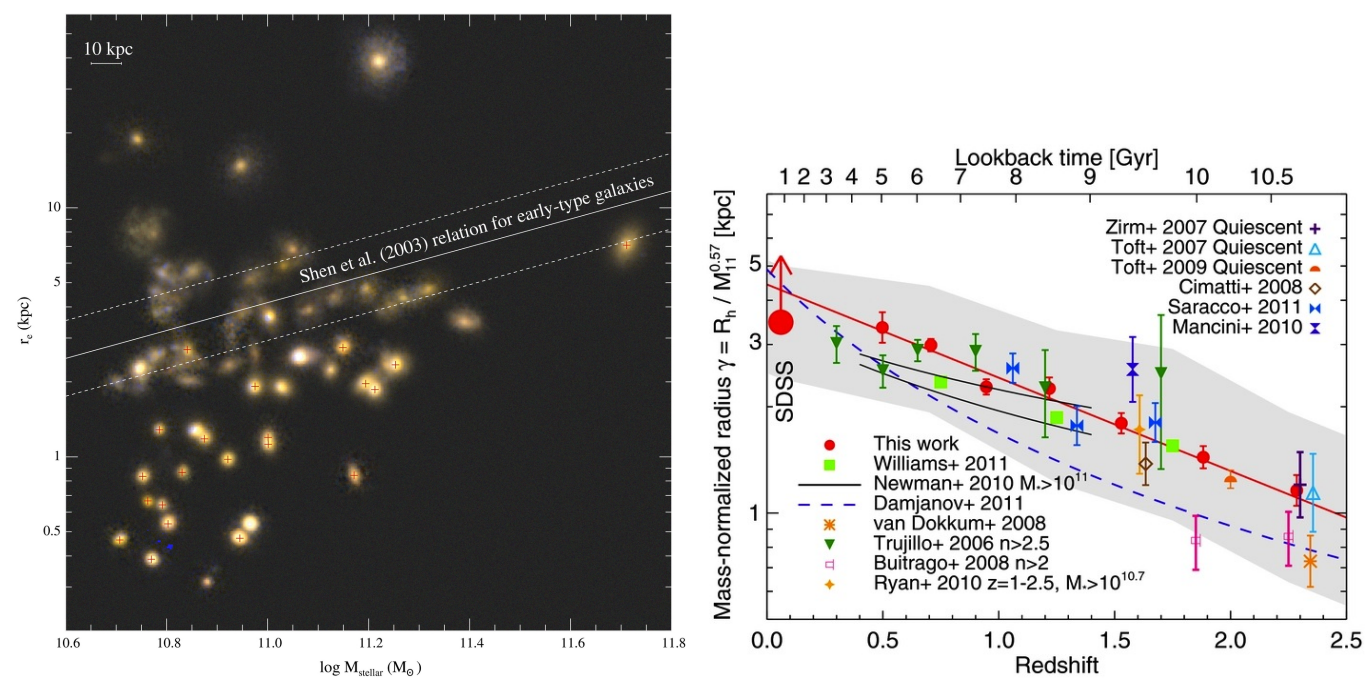

Figure 2: Left: Color images of all massive galaxies in the CANDELS GOODS-S field in size-mass space. Quiescent galaxies are marked with red crosses and are offset significantly from the $z \sim 0$ size-mass relation [41] for early type galaxies, from Szomoru et al. [43]. Right: Mass-normalized sizes indicate that the size evolution of massive galaxies occurs smoothly with redshift, compilation of observational results from Newman et al. [31].

\subsection{Unexpected and undeniable size evolution of massive galaxies}

An exciting observational result of the last decade is the fact that the past 10 billion years have been an active period in the lives of massive galaxies. The first evidence of this was the result that at high redshift massive galaxies are systematically more compact than their local counterparts at fixed mass [e.g. 15, 47, 50, 59, 69, and many subsequent papers]. The compactness of $z \sim 2$ galaxies in the GOODS-South CANDELS [21,25] field is shown graphically in the left panel of Figure 2, from Szomoru et al. [43]. Here, images of all massive galaxies are shown, sorted by size and stellar masses. Quiescent galaxies are marked with crosses, which can be compared to the labeled Shen et al. [41] mass-size relation for early type galaxies in the local universe. This observed size evolution has been found to occur smoothly over time (the right panel of Figure 2, 
from Newman et al. [31]), also measured with CANDELS [21, 25] imaging, in the GOODS-S and UKIRT UDS fields.

Several groups have looked for similarly dense and massive galaxies in the local universe $[44,49]$, finding that such galaxies are extremely uncommon today. In combination of the striking discrepancy between high-z galaxies and their local descendants, this dearth of massive compact relics implies that the structures of massive galaxies must have evolved strongly in the last 10 billion years. The way in which their structures have evolved can be used to constrain the physical mechanisms that primarily drive massive galaxy evolution, even after their star formation has ceased.

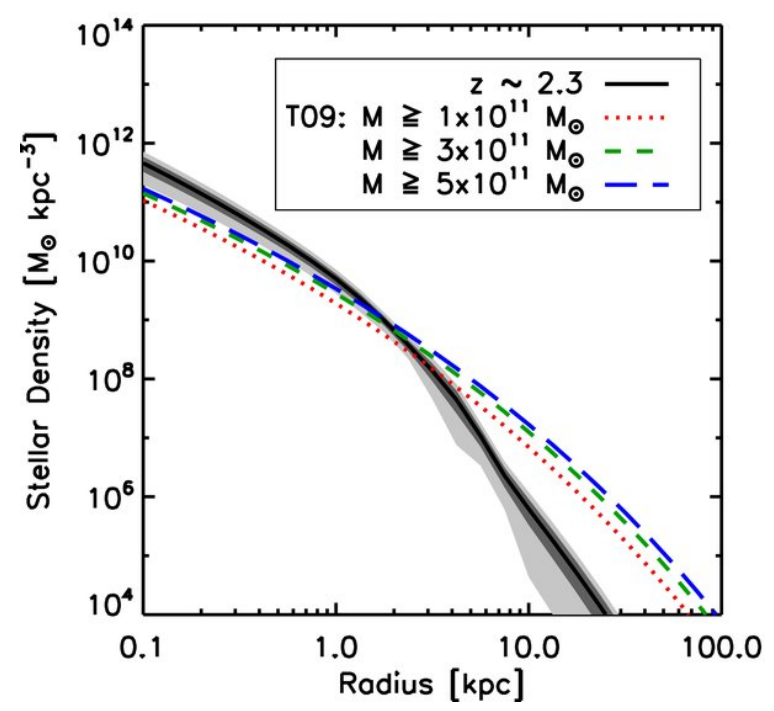

Figure 3: Density profiles of massive galaxies at $z \sim 2$ (gray) and $z \sim 0$ (colored lines). Although the overall sizes of the galaxy samples differ by a factor of $\sim 5$, the density within the central cores (within a physical aperture of $1 \mathrm{kpc}$ ) have remained roughly stable with time, from Bezanson et al. [7].

Observations of the density profiles of massive galaxies at high and low redshift have demonstrated that size evolution is primarily driven by growth at larger radii; the density in the central $\sim 1 \mathrm{kpc}$ of massive galaxies at high-redshift and local elliptical galaxies are quite similar (see Figure 3, [7]). In other words, in the last 10 billion years, massive galaxies have grown in an "inside-out" manner. Several physical mechanisms have been proposed to explain the structural evolution of massive galaxies. Most of these growth mechanisms require that individual galaxies grow in mass, requiring slightly more sophisticated methods, as opposed to evolution of net properties at fixed mass, to connect galaxy populations at different redshifts. The following subsections will discuss both new observational techniques, additional evidence for the evolution massive galaxies, and initial interpretations of these findings.

\subsection{A new technique to follow massive galaxies through cosmic time}

Comparisons of the properties of galaxies at fixed mass, as described in the previous section, have two major limitations. First, this type of study can only describe the evolution of large samples of galaxies, but does not trace the mass growth of individual galaxies, or populations of galaxies. 

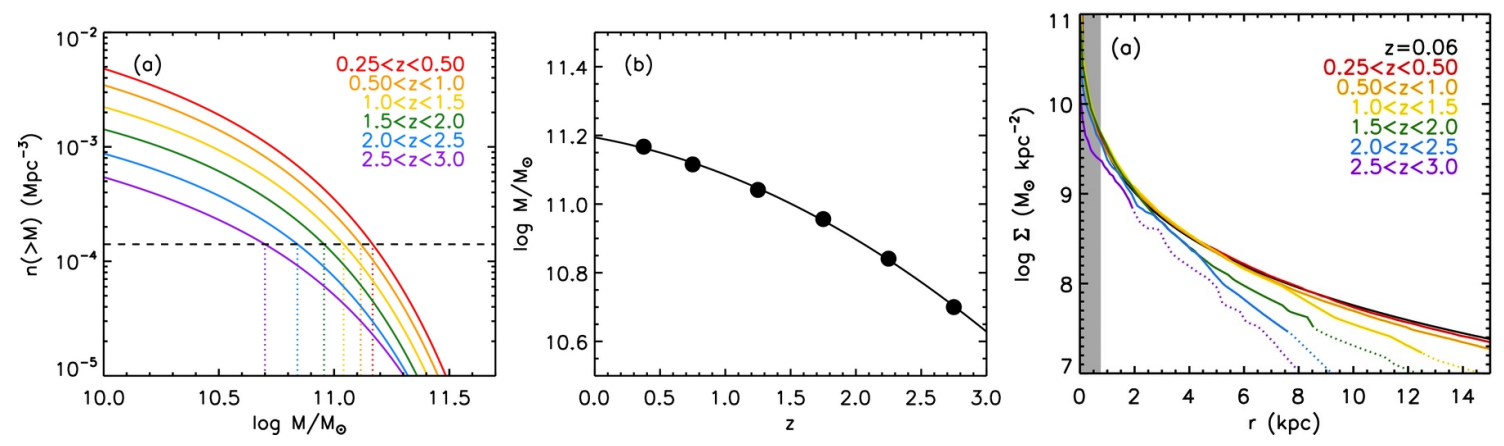

Figure 4: Left: Cumulative number density of galaxies as a function of stellar mass (cumulative stellar mass function). Horizontal dashed line indicates the number density $\left(2 \times 10^{-4} \mathrm{Mpc}^{-3}\right)$ at which galaxies are selected. Center: Mass growth of galaxies at $n(>M)=2 \times 10^{-4} \mathrm{Mpc}^{-3}$ as a function of redshift. Right: Density profile evolution at fixed number density. All figures from Patel et al. [37].

Secondly, the comparison of quiescent galaxies to quiescent galaxies at different epochs can introduce a "progenitor bias", in which a sample misses progenitor galaxies that do not yet fall into the sample parameters. In this particular instance, samples can preferentially exclude galaxies that have yet to quench their star-formation, but will eventually evolve into the population in question.

In an effort to better connect galaxy populations observed at different epochs, the technique of following galaxies at fixed cumulative number density, as defined by the stellar mass function, was developed [see e.g. 4, 28, 36, 37, 60]. In this methodology, one assumes that galaxies grow while maintaining approximate rank-order. Therefore, the growth of the stellar mass function within a bin in number density will describe the mass growth of galaxies within that sample (left and center panels of Figure 4). Furthermore, once the mass growth is quantified, one can study the evolution of this sample without the unphysical assumption of fixed mass comparisons. For example, one can trace the stacked density profiles of galaxies at fixed number density, as opposed to fixed mass, as shown in the right panel of Figure 4 [37]. The promise of this technique has grown dramatically as a result of vast improvements in the depths of photometric surveys.

\subsection{Structural Evolution of Massive Galaxies - Were they more disk-like?}

The observed inside-out growth of massive galaxies implies that their size growth is combined with a smooth morphological transformation. It has been suggested that massive galaxies at high redshifts are more disk-like than their local elliptical counterparts [e.g. 13, 54, 63]. These claims are largely based on the observed evolution of the shape of galaxy profiles, as quantified by bestfit Sérsic profiles. The Sérsic profile is a flexible $\rho \propto r^{1 / n}$ functional form that allows for more flexibility than the bimodal exponential disk $(n=1)$ and de Vaucouleurs $(n=4)$ profiles used to fit either disk or bulge components in local galaxies. By $z \sim 2$, the Sérsic indices of massive galaxies most resemble disk galaxies in the local universe (see e.g. the right panel of Figure 4 and the top row of Figure 5). However, evidence that these galaxies are clear analogs of local disks is not clear. Although their light profiles are similar, the distribution of ellipticities of $z \sim 2$ massive galaxies differ from those predicted for randomly oriented disks (bottom row of Figure 5, [13]).

Another test of the composition of massive, high-redshift galaxies is to measure their dynamics and compare the relationship between their measured structures and dynamics, as quantified by 

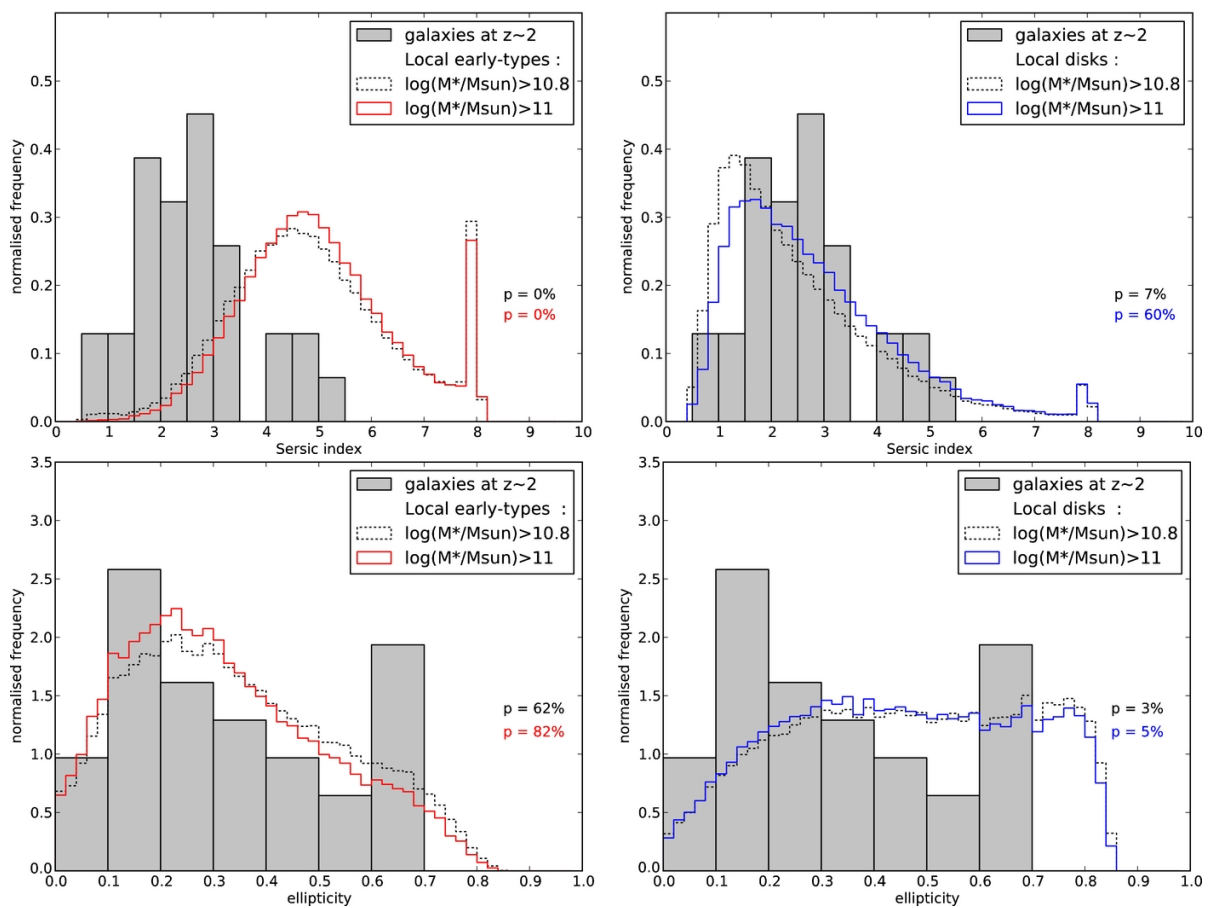

Figure 5: Sérsic index and ellipticity distributions of $z \sim 2$ compared to local early-type (left) and latetype (right) galaxies. The Sérsic indices of $z \sim 2$ galaxies most resemble local disk-like galaxies, while the ellipticities most resemble elliptical galaxies in the SDSS. From Chevance et al. [13].

their velocity dispersions. This can be used as an additional test of the galaxy compactness - both of the stellar mass and size estimates; the size and mass of a galaxy is related to the motion of the stars within that galaxy by the Virial theorem:

$$
\sigma \propto \sqrt{M / R}
$$

Measurements of galaxy velocity dispersions at $z \gtrsim 1$ require high signal-to-noise spectroscopy with at least medium resolution, which are only possible with extremely long ( $\sim 10$ hour) integrations on 8-10-meter-class telescopes. Despite the observational difficulty, such measurements have been made for $\sim 10-20$ massive galaxies at $z \gtrsim 1.5$ [e.g. 6, 32, 34, 46, 51, 52, 57]. The velocity dispersions of these galaxies appear to be consistent with those inferred by their compactness [e.g. $6,9,51,52,57]$.

Further complicating the question of whether massive galaxies were more disk-like is the observation that the mass fundamental plane, or the relation between stellar mass density, velocity dispersion, and sizes of massive galaxies is remarkably stable with time, without strong evidence for an evolution in the Virial relation [8] (see Figure 6). Theoretically, the constant of proportionality in the Virial equation will depend on the distribution of mass in a galaxy, therefore the structural evolution of massive galaxies would predict an evolution in this equation. However the relation between galaxy structures and dynamics for these galaxies does not show strong evidence for such evolution. In this context, the evolution of the mass fundamental plane reveals a simplicity in the evolution of massive galaxies since $z \sim 2$, despite their otherwise busy adolescence. 

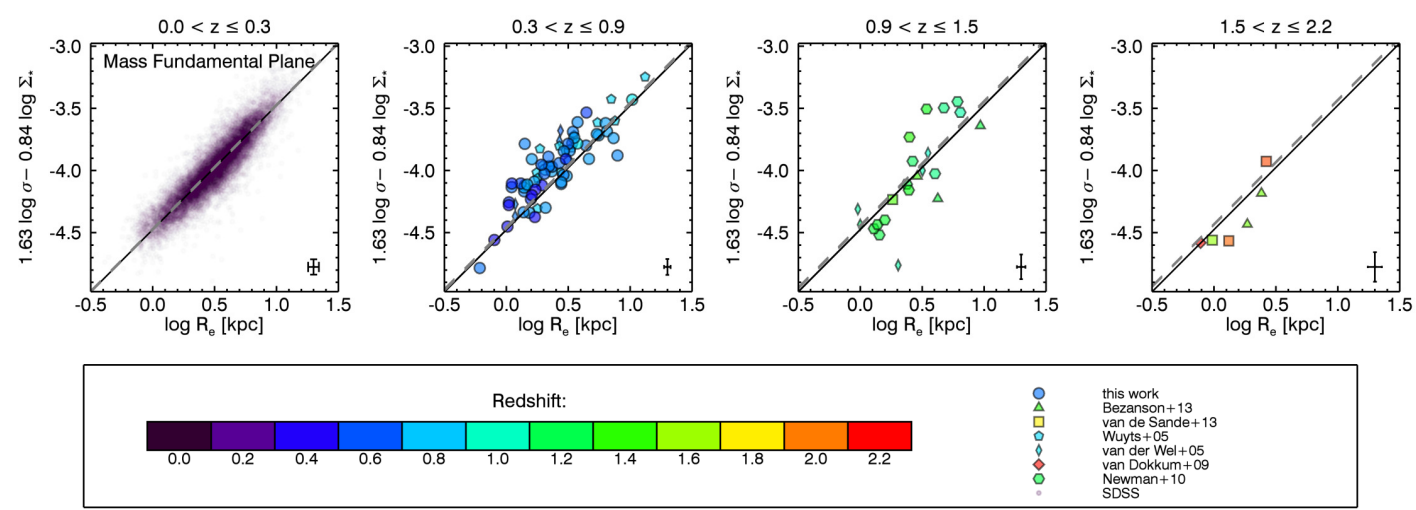

Figure 6: The mass fundamental plane as a function of redshift for massive galaxies from $0<z<2.5$, from Bezanson et al. [8]. The stability of this relation between galaxy sizes, masses and dynamics suggests that evolution occurs within the mass fundamental plane, without any strong evidence for significant dynamical evolution, such as an increased importance of rotational support.

However, this simple relation may be obscuring an underlying complexity due to size evolution (and inside-out morphological growth), possibly evolving dark matter fractions, uncertain and potentially non-uniform initial mass functions, observational uncertainties, and finally quenching and changing galaxy populations, to name a few.

\subsection{Star-formation and quenching: not all massive galaxies were red and dead}

Although locally, massive galaxies are primarily red and dead, high redshift observations begin to probe eras during which many of these galaxies were still forming stars or had recently quenched their star-formation. By $z \sim 2$, approximately half of massive $\left(M_{*} \sim 10^{11} M_{\odot}\right)$ galaxies were still star-forming [e.g. 10, 29]. These massive star-forming galaxies are largely forming stars at a very high rate, comprising the high-mass tip of the star formation rate (SFR)-stellar mass relation, or "star-forming main sequence", that appears to exist out to at least $z \sim 2.5$ [e.g. 65, 68].

It is extremely rare to catch a massive galaxy in the process of quenching - that is when it is still forming stars, but has a SFR far below the star-forming main sequence. Therefore, quenching is an apparently rapid process. Many of the galaxies designated as quiescent at $z \gtrsim 1$ show signs of recent quenching, both in their colors [e.g. 64] and spectroscopically [e.g. 6, 35]. In fact, many massive quiescent galaxies at $z \sim 1.5$ show strong Balmer lines, which indicate that they must have been strongly star-forming in the past, with the most recent burst of star-formation quenched $<1$ Gyr prior to the observations [e.g. 6, 35].

Much like their local counterparts, star-forming and quiescent galaxies are structurally different at high-redshift. On average, massive star-forming galaxies are a factor of $\sim 2$ larger than quiescent galaxies of the same mass, at nearly all redshifts [e.g. 27, 31, 67]. It is important to emphasize, however, that all size-mass relations demonstrate significant scatter and individual compact galaxies with on-going star-formation have been observed [e.g. 2,3]. Given the evolving quenched fraction and structural differences, one must incorporate the evolution in the composition of the massive galaxy population with time in explaining its observed growth. 


\subsection{Explaining the evolution of massive galaxies}

The past $\sim 10$ billion years have seen significant evolution in the physical properties of massive galaxies. They have evolved structurally and many have undergone the quenching of star formation. However, the physical mechanisms that are involved in causing this evolution, particularly in the galaxy size and morphologies, are still debated.

With respect to the observed size evolution of massive, quiescent galaxies, the leading model involves significant minor merging. There is very little or no star formation in these galaxies and therefore cannot be responsible for their growth. Major mergers of galaxies, defined by mass ratios greater than $\sim 1: 4$, could also increase galaxy sizes. However, the addition of large stellar bodies would also largely increase the masses of massive galaxies, overproducing extremely massive galaxies with respect to the overall evolution of the stellar mass function [e.g. 7]. Minor merging, on the other hand can efficiently increase galaxy sizes without adding significant mass [e.g. 7, 30].

To first order, the prevalence of minor merging and its ability to grow galaxies "inside-out" by adding stars from the satellite galaxies to the galaxy outskirts has been demonstrated in cosmological simulations [e.g. 22, 30]. Furthermore, although massive galaxies are primarily regular and undisturbed, many have been observed, particularly at higher redshifts, to show significant signs of ongoing interactions, such as tidal features, shells and streams [e.g. 55, 56]. In fact, in a volumelimited imaging survey of low surface brightness features in local massive ellipticals, $\sim 70 \%$ of galaxies showed deviations from smooth Sérsic light profiles.

On the other hand, the amount of minor merging required to produce the factor of $\sim 3-5$ size growth in these galaxies is hard to constrain, and may not be consistent with merger rates or the observed satellite populations around massive galaxies. One study of satellite galaxies surrounding massive quiescent galaxies found that while the number of satellites could be sufficient to reproduce the size evolution at $z \lesssim 1$, there were too few satellite galaxies to explain the growth at higher redshifts $(1 \lesssim z \lesssim 2.5)$ [32].

Another suggestion has been that the size evolution is, at least in part driven by the evolution in galaxy populations [e.g. 12, 19, 39, 53, 59]. Star forming galaxies have larger average sizes, therefore, if the process that truncates star formation does not alter their structures, the quenching of star forming galaxies would increase the average size of quiescent galaxies with time. Although this is likely occurring, recent evidence suggests that it cannot be the only mechanism driving the size growth of massive galaxies. First, one study showed that the sizes of younger (bluer) quenched galaxies are consistent with the average sizes of older galaxies at $z \gtrsim 1$ [64]. Furthermore, although studies of the size evolution of quiescent galaxies are sensitive to the effects of quenching, the entire massive galaxy population is not sensitive to this progenitor bias (as defined by number density selection, for example) shows overall size evolution as well [e.g. 37, 60].

\section{Conclusions}

Overall, recent studies have found that the lives of massive galaxies since $z \sim 2.5$ have been quite eventful, perhaps significantly more than their relaxed morphologies and dynamics and old stars today would suggest. Substantial improvements in extragalactic pencil-beam surveys, nearIR detector technologies, spectroscopic breakthroughs, and ingenuity in discovery and analysis techniques have contributed dramatically to this field. 
In the future, our understanding of the lives of massive galaxies, and even lower mass galaxies, will continue to grow with bigger samples and to higher redshifts, with the advent of better NIR spectrographs, space-based JWST, and eventually 30-meter class telescopes. We can continue to try to explain the morphological evolution of massive galaxies and formation of massive galaxies. We will discover how such compact galaxies are able to form at $z \gtrsim 2$ and explore the properties of their progenitors. We will disentangle seemingly conflicting evidence about the possible disk-like nature of $z \sim 2$ compact galaxies.

For example, with large telescopes, adaptive optics, and an excellent NIR spectrograph, one could attempt to resolve their rotation and therefore confirm whether the dynamics of compact massive galaxies are consistent with local dislike morphologies. With the combination of observations of progenitor galaxies at earlier epochs and strong observational constraints on the structures and dynamics of massive galaxies we will be able to explore the formation mechanisms for these compact galaxies. Whether their stars follow organized disk-like rotation or are dispersion dominated like local ellipticals will place strong constraints on these formation mechanisms.

Finally, although this article focused on the evolution of massive galaxies, high-redshift galaxy studies are increasingly able to follow galaxy populations through cosmic time at lower and lower masses. Recent studies have begun to extend this work to galaxies at the mass of the Milky Way [e.g. 38, 61]. This work will continue to disentangle the observational evidence and improve our understanding of how galaxies of all sizes evolve in the Universe.

\section{References}

[1] Abazajian, K., et al. 2003, The Astronomical Journal, 126, 2081

[2] Barro, G., et al. 2013, The Astrophysical Journal, 765, 104

[3] —. 2013, arXiv:1311.5559

[4] Behroozi, P. S., et al. 2013, The Astrophysical Journal Letters, 777, L10

[5] Bell, E. F., et al. 2004, The Astrophysical Journal, 608, 752

[6] Bezanson, R., et al. 2013, The Astrophysical Journal Letters, 764, L8

[7] —. 2009, The Astrophysical Journal, 697, 1290

[8] —. 2013, The Astrophysical Journal Letters, 779, L21

[9] —. 2011, The Astrophysical Journal Letters, 737, L31+

[10] Brammer, G. B., et al. 2011, The Astrophysical Journal, 739, 24

[11] —. 2012, The Astrophysical Journal Supplement, 200, 13

[12] Carollo, C. M., et al. 2013, The Astrophysical Journal, 773, 112

[13] Chevance, M., et al. 2012, The Astrophysical Journal Letters, 754, L24 
[14] Coil, A. L., et al. 2011, The Astrophysical Journal, 741, 8

[15] Daddi, E., et al. 2005, The Astrophysical Journal, 626, 680

[16] Davis, M., et al. 1982, The Astrophysical Journal, 253, 423

[17] de Vaucouleurs, G., et al. 1991, Third Reference Catalogue of Bright Galaxies. Volume I: Explanations and references. Volume II: Data for galaxies between $0^{h}$ and $12^{h}$. Volume III: Data for galaxies between $12^{h}$ and $24^{h}$.

[18] Förster Schreiber, N. M., et al. 2006, The Astronomical Journal, 131, 1891

[19] Franx, M., et al. 2008, The Astrophysical Journal, 688, 770

[20] —. 2003, The Astrophysical Journal Letters, 587, L79

[21] Grogin, N. A., et al. 2011, The Astrophysical Journal Supplement, 197, 35

[22] Hilz, M., Naab, T., \& Ostriker, J. P. 2012, ArXiv e-prints

[23] Hu, E. M., Cowie, L. L., \& McMahon, R. G. 1998, The Astrophysical Journal Letters, 502, L99+

[24] Illingworth, G. D., et al. 2013, The Astrophysical Journal Supplement, 209, 6

[25] Koekemoer, A. M., et al. 2011, The Astrophysical Journal Supplement, 197, 36

[26] —. 2013, The Astrophysical Journal Supplement, 209, 3

[27] Law, D. R., et al. 2012, The Astrophysical Journal, 745, 85

[28] Leja, J., van Dokkum, P., \& Franx, M. 2013, The Astrophysical Journal, 766, 33

[29] Muzzin, A., et al. 2013, The Astrophysical Journal, 777, 18

[30] Naab, T., Johansson, P. H., \& Ostriker, J. P. 2009, The Astrophysical Journal Letters, 699, L178

[31] Newman, A. B., et al. 2012, The Astrophysical Journal, 746, 162

[32] Newman, A. B., et al. 2010, The Astrophysical Journal Letters, 717, L103

[33] Newman, J. A., et al. 2012, arXiv:1203.3192

[34] Onodera, M., et al. 2010, The Astrophysical Journal Letters, 715, L6

[35] —. 2012, The Astrophysical Journal, 755, 26

[36] Papovich, C., et al. 2011, Monthly Notices of the Royal Astronomical Society, 412, 1123

[37] Patel, S. G., et al. 2013, The Astrophysical Journal, 766, 15

[38] —. 2013, arXiv:1304.2395 
[39] Poggianti, B. M., et al. 2013, The Astrophysical Journal, 762, 77

[40] Quadri, R., et al. 2007, The Astronomical Journal, 134, 1103

[41] Shen, S., et al. 2003, Monthly Notices of the Royal Astronomical Society, 343, 978

[42] Steidel, C. C., et al. 1996, The Astrophysical Journal Letters, 462, L17

[43] Szomoru, D., Franx, M., \& van Dokkum, P. G. 2012, The Astrophysical Journal, 749, 121

[44] Taylor, E. N., et al. 2010, The Astrophysical Journal, 720, 723

[45] Tilvi, V., et al. 2013, The Astrophysical Journal, 768, 56

[46] Toft, S., et al. 2012, The Astrophysical Journal, 754, 3

[47] —. 2007, The Astrophysical Journal, 671, 285

[48] Tomczak, A. R., et al. 2013, arXiv:1309.5972

[49] Trujillo, I., et al. 2009, The Astrophysical Journal Letters, 692, L118

[50] —. 2006, The Astrophysical Journal, 650, 18

[51] van de Sande, J., et al. 2011, The Astrophysical Journal Letters, 736, L9

[52] —. 2013, The Astrophysical Journal, 771, 85

[53] van der Wel, A., et al. 2009, The Astrophysical Journal Letters, 706, L120

[54] —. 2011, The Astrophysical Journal, 730, 38

[55] van Dokkum, P. G. 2005, The Astronomical Journal, 130, 2647

[56] van Dokkum, P. G., \& Brammer, G. 2010, The Astrophysical Journal Letters, 718, L73

[57] van Dokkum, P. G., Kriek, M., \& Franx, M. 2009, Nature, 460, 717

[58] van Dokkum, P. G., et al. 2003, The Astrophysical Journal Letters, 587, L83

[59] —. 2008, The Astrophysical Journal Letters, 677, L5

[60] —. 2010, The Astrophysical Journal, 709, 1018

[61] —. 2013, arXiv:1304.2391

[62] Vogt, N. P., et al. 2005, The Astrophysical Journal Supplement, 159, 41

[63] Weinzirl, T., et al. 2011, The Astrophysical Journal, 743, 87

[64] Whitaker, K. E., et al. 2012, The Astrophysical Journal, 745, 179

[65] —. 2012, The Astrophysical Journal Letters, 754, L29 
[66] Whitaker, K. E., et al. 2011, The Astrophysical Journal, 735, 86

[67] Williams, R. J., et al. 2009, The Astrophysical Journal, 691, 1879

[68] Wuyts, S., et al. 2011, The Astrophysical Journal, 742, 96

[69] Zirm, A. W., et al. 2007, The Astrophysical Journal, 656, 66 